\title{
Myocardial Ischemia Following Shrapnel Epicardiac Injury 16 Years Earlier: Case Report
}

\author{
Jaffar S. Shehatha1,2, Dara M. Mohialdeen ${ }^{3}$, Abdulsalam Y. Taha ${ }^{4}$ \\ ${ }^{1}$ Cardiac Surgery Department, PAR Hospital, Irbil, Iraq \\ ${ }^{2}$ School of Surgery, Western Australia University, Perth, Australia \\ ${ }^{3}$ Department of Cardiology, Sulaimania Centre for Heart Disease, Sulaimania, Iraq \\ ${ }^{4}$ Department of Cardiothoracic and Vascular Surgery, School of Medicine and Sulaimania Teaching Hospital, \\ Sulaimania, Iraq \\ Email:
}

Received 8 December 2013; revised 7 January 2014; accepted 5 February 2014

Copyright (C) 2014 by authors and Scientific Research Publishing Inc.

This work is licensed under the Creative Commons Attribution International License (CC BY).

http://creativecommons.org/licenses/by/4.0/

\section{(c) (i) 0 pen Access}

\begin{abstract}
Coronary artery injuries are uncommon. Acute injuries of major coronary vessels, particularly the left anterior descending artery (LAD), are life-threatening because of the significant cardiac dysfunction that ensues and usually requires repair or bypass. In contrast, late presentation of missile coronary artery injuries has been sporadically reported in the literature. Herein we present a 32year-old lady from Sulaimania, Iraq with multiple shrapnel injuries 16 years earlier presented recently with severe anterolateral myocardial ischemia unresponsive to medical treatment. Coronary angiography and surgical exploration revealed shrapnel compressing the LAD. The patient responded very well to shrapnel extraction and coronary artery revascularization (off pump left internal mammary artery-LIMA-to LAD anastomosis). The management of this patient is discussed with review of relevant literature. It is concluded that long-standing shrapnel close to a major coronary artery may incur an external trauma initiating a localized arteriosclerosis with an extremely long time interval from injury to the onset of symptoms of myocardial ischemia.
\end{abstract}

\section{Keywords}

Coronary Artery; Late Injuries; Epicardiac; Myocardial Ischemia; Repair; Revascularization

\footnotetext{
${ }^{*}$ Corresponding author.
} 


\section{Introduction}

Coronary artery injuries are uncommon [1] [2]. The reported incidence is $3.1 \%$ to $4.4 \%$ of penetrating cardiac injuries [3]. Acute injuries of major coronary vessels particularly the LAD are life-threatening because of the significant cardiac dysfunction that ensues and usually requires repair or bypass [1]. In contrast, missile coronary artery injuries with late presentation (years or even decades after the onset of trauma) are rare [4] [5]. Sporadic similar cases were previously reported [5]. Herein, we present a case of coronary artery compression attributed to shrapnel injury 16 years earlier. The case is discussed with literature review looking for possible explanation of this late presentation.

\section{Clinical Summary}

A 32 years old lady, a farmer from Sulaimania, Iraq was admitted to Sulaimania Centre for Heart Disease (SCHD) on November 27, 2010 because of severe chest pain radiating to her lower jaw of few days duration. The pain could not be relieved by analgesic drugs given to her by a local doctor. She was non-smoking and had no family history of heart disease or hypertension and had a normal lipid profile. The obstetric and gynaecological history was unremarkable (she was married at the age of 20, had 5 normal pregnancies and became a mother of 5 healthy children). She was exposed to a mine explosion 16 years earlier (when she was a girl of 16). She had sustained multiple body injuries (head, chest and extremities) described by a medical report as being superficial, thus responded well to conservative treatment. Her marriage followed the injury by 4 years. She had a normal health until recently.

In-patient investigations included electrocardiography (ECG) which showed Q and T-waves inversion in V1-V6 chest leads and a positive (qualitative) troponin test; both were consistent with anterolateral myocardial ischaemia. The plain chest radiograph (lateral view) showed multiple small shrapnels anterior to the heart. An urgent coronary angiography (Figures 1(a)-(c)) was arranged. It displayed a compression of the second part of LAD by shrapnel.

Meanwhile, the patient received a medical treatment for acute myocardial ischaemia in the coronary care unit (CCU) in form of heparin and intravenous nitro-glycerine infusion. Despite this intensive medical therapy, the chest pain was increasing in severity. The cardiac surgeon was consulted and the decision of surgical intervention was made.

The patient was approached via median sternotomy. LIMA was harvested in the standard manner. Facilities to go immediately on cardio-pulmonary bypass were made ready in case this was needed. After opening the pericardium (which was surprisingly normal), sharp dissection was made in the dense fibrous tissue anterior to the heart; the site of previous trauma. An irregular sharp shrapnel $2 \times 2.5 \mathrm{~cm}$ in size was found and extracted; there was no direct injury but compression of LAD.

Revascularization was then achieved by LIMA to LAD anastomosis on a beating heart. The postoperative course was uneventful; the patient left the hospital on the 5th postoperative day.

Follow-up of this patient showed that the chest pain never returned, she was not in need of anti-angina medication though she resumed her job as a housewife and a farmer doing heavy physical activities.

\section{Comment}

Penetrating cardiac injuries which result in laceration of coronary arteries usually present acutely in the form of bleeding and acute myocardial ischemia. Urgent operation is usually required for the sake of bleeding. Coronary revascularization in the emergency setting depends on the available facilities and the circumstances of the individual case. Al Museilih reported on a 16-year-old girl with a stab wound to the heart presented with massive left haemothorax and coronary artery injury [4]. She was saved by an emergency thoracotomy for the haemothorax and suturing of the ventricular wound. She survived the operation but had acute inferolateral myocardial ischemia. She did well for about 6 years but significant angina developed later which required subsequent coronary revascularization. In contrast, H. M. Chin et al., described LAD artery incomplete laceration in 32-year-old man due to pneumatic nail gun; the arterial injury was repaired directly without cardiopulmonary bypass and the nail was also removed [2].

When ischaemic heart disease is considered, the pathologic lesion responsible for myocardial ischemia is commonly atherosclerosis with or without superadded thrombosis. The risk factors for atherosclerosis are well 

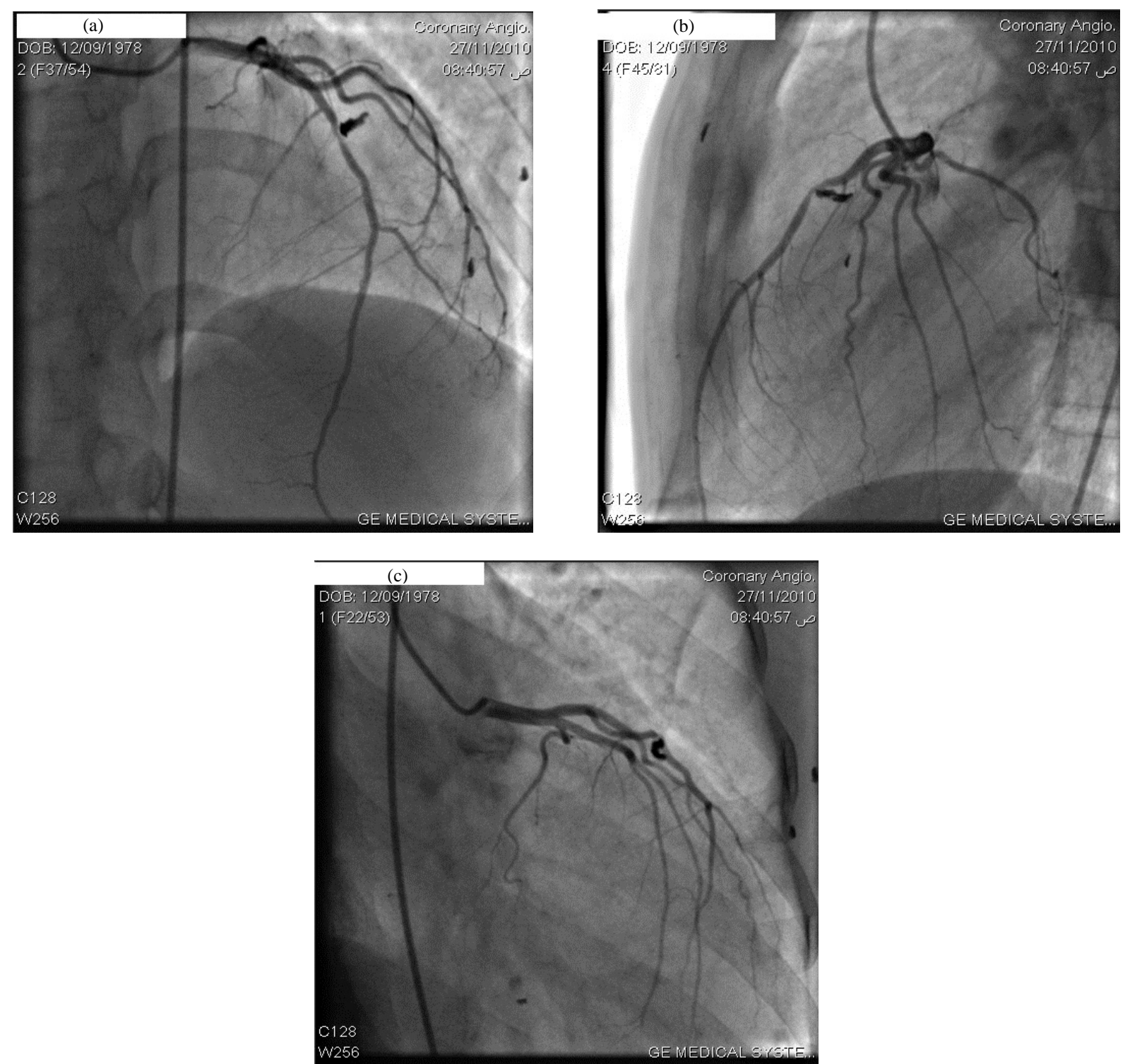

Figure 1. (a) Coronary angiogram (Antero-posterior view) revealing sharp shrapnel compressing LAD artery; (b) Lateral view; (c) Oblique view.

known to every physician and include ageing, smoking, hypertension, diabetes, hyperlipidaemia and restricted physical activity. Looking at our patient, we see that almost she lacked any of these predisposing factors. She worked as a farmer with heavy physical daily activities, was non-smoking, normotensive, euoglycaemic with normal lipid profile. Therefore, it is unlikely to link the myocardial ischemia in our patient with atherosclerosis. The logic sequence of thinking is to accuse trauma as the location of the shrapnel seen by plane x-ray and coronary angiography was very close to LAD, the artery in which myocardial ischemia occurred in its territory. But how can we explain the long latency period between the date of injury and onset of symptom? Why this patient was symptom-free for almost 16 years? Was there a recent migration of the shrapnel that caused compression of the coronary artery and occurrence of symptoms? Well, with the dense fibrous tissue usually induced by the presence of a foreign body for such a long time in the epicardium, this hypothesis seems illogic.

Faced with this interesting case for which we could not find a ready answer; we ought to search the literature. To our surprise, the first article we got in our search for (missile epicardiac or coronary artery injuries) was one written by Seipelt et al. from Germany reporting on 72-year-old man who was injured in 1943 during World War II, by fragments from an explosive Russian device, then recovered completely. The man developed atypical 
angina 44 years following the initial injury. Investigations revealed 2 shrapnels; the first had caused stenosis of right coronary artery while the second compressed the LAD. Both fragments were retrieved surgically besides revascularization of both arteries [5]. The authors found atherosclerotic plaque built in the area of arterial stenosis [5]. According to Seipelt et al., Shirani and colleagues in 1994 published a similar case of a bullet located in the epicardium adjacent to the left main coronary artery causing death of a patient 24 years after the injury and discovered only at autopsy [5]. Bland and Beebe reviewed the World War II surgical experience and found that most patients with foreign bodies in and around the heart were asymptomatic at 20-year follow-up [6]. It is postulated that the permanent pulsation of the vessel against the adjacent missile and the consequent turbulence in the blood flow result in building up of an atherosclerotic plaque in the area of the artery giving origin to the stenosis [5]. Our case developed symptoms of myocardial ischemia 16 years after the injury; a time needed to develop a localized arterial stenosis according to the aforementioned hypothesis.

\section{Conclusion}

External trauma to the major epicardial coronary arteries may initiate arteriosclerosis which can be limited exclusively to the area of injury. The time frame from injury to the onset of symptoms can be extremely long [5].

\section{Conflict of Interest}

There is no conflict of interest.

\section{References}

[1] Irwin, R.S. and Rippe, J.M. (2008) Intensive Care Medicine. 6th Edition, Lippincott Williams \& Wilkins, Philadelphia, 402. http://books.google.iq/books?isbn=0781791537

[2] Chen, H.-M., Chou, C.-J., Shih, M.-C.P. and Yeh, Y.-S. (2011) An Unusual Fatal Penetrating Coronary Artery Injury with Effective Perioperative Management: Case Report. European Journal of Cardio-Thoracic Surgery. (in Press)

[3] Mayo, G.L. (2013) Penetrating Cardiac Trauma. (Internet Monograph) http://www.medicine.mcgill.ca/mjm/issues/v01n01/cardiac.html

[4] Taha, A.Y. (2005) Emergency Thoracotomy for Cardiac or Great Vessel Injuries: A Report of 5 Cases. Basrah Journal of Surgery, 11, 8p.

[5] Seipelt, R.G., Vazquez-Jimenez, J.F. and Messmer, B.J. (2000) Missiles in the Heart Causing Coronary Artery Disease 44 Years after Injury. The Annals of Thoracic Surgery, 70, 979-980. http://dx.doi.org/10.1016/S0003-4975(00)01672-6

[6] Bland, E.F. and Beebe, G.W. (1966) Missiles in the Heart: A 20-Year Follow-Up Report of World War II Cases. New England Journal of Medicine, 274, 1039-1046. http://dx.doi.org/10.1056/NEJM196605122741901 